\title{
Surfaces
}

\section{LOST IN SPACE. QUELQUES CONCEPTS CLÉS DE LA THÉORIE QUEER}

\section{Eric Dupont}

Volume 3, 1993

URI : https://id.erudit.org/iderudit/1065105ar

DOI : https://doi.org/10.7202/1065105ar

Aller au sommaire du numéro

\section{Éditeur(s)}

Les Presses de l’Université de Montréal

\section{ISSN}

1188-2492 (imprimé)

1200-5320 (numérique)

Découvrir la revue

\section{Citer cet article}

Dupont, E. (1993). LOST IN SPACE. QUELQUES CONCEPTS CLÉS DE LA THÉORIE QUEER. Surfaces, 3. https://doi.org/10.7202/1065105ar
Résumé de l'article

Compte-rendu thématique de Queer Sites : Bodies at Work, Bodies at play, colloque d'études culturelles lesbiennes et gaies tenu à Toronto en mai 1993. L'auteur discute les diverses notions d'espace et de positionnement discursif véhiculées ou thématisées lors de la rencontre. Il désigne ensuite la littérature comme lieu d'élaboration possible d'un espace véritablement queer.
Copyright ( $\odot$ Eric Dupont, 1993

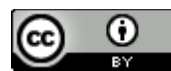

Ce document est protégé par la loi sur le droit d'auteur. L'utilisation des services d'Érudit (y compris la reproduction) est assujettie à sa politique d'utilisation que vous pouvez consulter en ligne.

https://apropos.erudit.org/fr/usagers/politique-dutilisation/ 


\title{
LOST IN SPACE
}

\section{QUELQUES CONCEPTS CLÉS DE LA THÉORIE QUEER}

Eric Dupont

\section{RÉSUMÉ}

Compte-rendu thématique de Queer Sites: Bodies at Work, Bodies at play, colloque d'études culturelles lesbiennes et gaies tenu à Toronto en mai 1993. L'auteur discute les diverses notions d'espace et de positionnement discursif véhiculées ou thématisées lors de la rencontre. Il désigne ensuite la littérature comme lieu d'élaboration possible d'un espace véritablement queer.

\begin{abstract}
Review of Queer Sites: Bodies at Work, Bodies at play, a lesbian and gay cultural studies conference held in Toronto in may 1993. The author discusses the notions of space and of discursive positionning as used or thematized by a number of participants. He then points to literature as a possible site for the working out of a truly queer space.
\end{abstract}

Le compte-rendu précédant[1] a sûrement servi à éclairer la lanterne des néophytes des théories queer, lesbienne, gay et autres créatures d'outreespace, et à mettre chacun au diapason de la rumeur montante qu'est la théorie queer dans le champ des études littéraires. Dans cet état de choses, 
j'aimerais attirer l'attention du lecteur sur quelques concepts chaudement discutés lors de cette rencontre internationale. Soit dit en passant, le titre de cet article, parce qu'il est et ne peut être qu'en anglais, exprime d'ores et déjà le ton des commentaires qui suivront à l'égard de la notion d'espace dans la théorie queer. Il s'agit de penser un lieu d'énonciation de la théorie queer, où l'homosexualité ne serait plus traitée comme le prolongement d'une essence et où l'expérience personnelle ne justifierait pas le cloisonnement des corps dans des catégories stables correspondant à un "espace" discursif.

Il m'a semblé que les concepts de position du sujet, d'énonciation et d'espace ont été inexorablement récurrents pendant le colloque. Et pour cause, ils constituent probablement la clef de voûte de tout le discours queer. Le rejet ou l'acceptation par les instances assignées à la production du discours théorique queer d'un consensus sur ces deux concepts pourraient fort bien décider de l'avenir et de la crédibilité du projet queer non seulement dans l'institution, mais au sein même du groupe humain qui forme son objet d'analyse. Avant de poursuivre, il est nécessaire d'effectuer un retour sur les débats théoriques à l'origine des réflexions qui vont suivre. Je me suis aussi permis, au cours de mes réflexions, de me servir d'éléments périphériques à la conférence. Des données telles la provenance des intervenants, le lieu géographique de la conférence et l'atmosphère générale de ces quatre jours de colloque m'apparaissent aussi importantes dans l'approche de l'objet que les arguments principaux des conférenciers. À titre d'exemple, l'Université de Toronto comme lieu queer officiel obligera le conférencier américain à adapter son discours à la réalité canadienne, surtout lorsqu'il s'agit d'une présentation concernant le rapport entre le système juridique et l'homosexualité. Voilà un déplacement auquel toute l'attention nécessaire n'a sûrement pas été portée. Le queer, comme disait Mary Bryson, pourrait finir par s'énoncer par et pour lui-même. Ou encore, par la mise en jeu d'une variable géographique supplémentaire à la restriction à un groupe sexuel: le discours anglo-américain queer s'énonce par et pour lui-même.

Mes préoccupations épistémologiques ont émergé au cours des discussions qui ont suivi les conférences suivantes: "Queer Pedagogy, An Unten(ur)able Discursive Posture" de Suzanne de Castell et Mary Bryson (University of British Columbia), "Against Foucault, or How I learned to Stop Worrying About and Start Loving Gay History" de Matt Kuefler (Yale University), "Espaces féministes et espaces queer" de Jeannelle Savonna (University of Toronto), "What colour are your Underwear?: Advertising Class and Whiteness in Gay Popular Imagery--An Illustrated Talk" de Steven Maynard (Queen's University), "Queer "Theory" Versus Heterosexual Hegemony: Towards a Historical Materialism for Queers" de Gary Kinsman ( Acadia University), et finalement "Notes from Camp Canada" de Andy Fabo, artiste de Toronto. Il ne s'agira pas ici de faire état des arguments principaux de chaque conférence, mais plutôt de localiser le lieu de croisement dans les discours des conférenciers. Ce lieu se situe selon toute apparence dans les concepts de positionnement du sujet et d'espace discursif, et on pourrait 
postuler qu'ils ressortent comme étant les prémisses de base de tout discours analytique du phénomène queer.

\section{Montrer patte blanche}

L'usage de termes géographiques comme outils d'analyse dans l'énonciation du discours queer était essentiel à l'argumentation de ces conférencières. Suzanne de Castell et Mary Bryson parlent de la position inconfortable du sujet lesbien qui enseigne un corpus de théorie queer ou féministe. À qui s'adressent ces cours et quelle est la position à adopter à l'intérieur de l'institution quant au dévoilement de son orientation sexuelle? Qui est en mesure d'inclure des ouvrages queer dans son corpus et à qui devrait-on l'enseigner? Doit-on s'adresser aux hétérosexuels en tant que lesbienne, /pp. 6-7/ ou réserver ce positionnement à un auditoire qui se trouve déjà dans l'espace queer et serait ainsi en meilleure posture pour séparer l'expérience personnelle du sujet parlant de la matière enseignée? La crédibilité et l'autorité au sein du discours queer seraient-elles mises en jeu par l'identité homosexuelle du sujet? Il y a lieu, en effet, de s'interroger sur ces questions tant et aussi longtemps que le sujet croit pouvoir se qualifier en tant qu'entité parlante de l'intérieur d'un certain espace auquel on accéderait par l'expérience homosexuelle. Une des questions soulevées pendant cette contribution fut celle du refus d'être considérée comme un cliché au sein de l'institution universitaire et d'être ainsi marginalisée comme un phénomène à part, en dehors du discours dominant. Il s'agit de démontrer à quel point la théorie queer conduit trop souvent à cette marginalisation du sujet dans un espace qu'il s'est lui-même défini. Il y a un point commun aux multiples utilisations des concepts d'espace et de positionnement du sujet: il s'agirait selon moi d'un essentialisme trop souvent utilisé inconsciemment dans le but de créer un espace discursif factice, basé sur un motif d'exclusion qui frise la théorie de la conspiration. Pour en revenir à l'exposé de Mary Bryson et de Suzanne de Castell, je dirai crûment que la question de savoir si le corps enseignant se doit de dévoiler son orientation sexuelle (quand le sujet est, selon toute apparence, un clone parfait de la construction iconographique de la lesbienne nord-américaine) fournit sa propre réponse. Je ne mets pas en question la capacité de l'académique homosexuel d'enseigner et de répandre dans l'institution ses préoccupations dans le champ précis des études queer, mais je dirais que son souci d'être en perte de crédibilité, due à son insistance sur sa position en tant qu'objet et sujet du discours, naît justement de ce recours à l'expérience personnelle en tant que justification de son lieu d'énonciation. Selon les deux conférencières, le seul chemin possible pour le développement de la théorie queer serait son énonciation par un sujet qui accepte l'essentialisme homosexuel et qui base, consciemment ou non, sa construction culturelle sur sa politique du désir. 
En guise de conclusion à leurs interrogations, elles ont avancé la suggestion de l'articulation du discours queer, par et pour eux-mêmes (elles-mêmes?). Cette déclaration de non- confiance à la population étudiante hétérosexuelle (c'est à dire, selon leur définition, des individus ne se réclamant pas d'une essence queer de par leurs expériences) est un appel à la ghettoïsation des études queer dans le sens que le sujet supposé hétérosexuel se voit interdit l'accès à un espace discursif basé sur l'annulation de la démarcation entre l'espace privé et public. C'est aussi attribuer des intentions discriminatoires a priori à un groupe d'étudiants qui ne pourraient faire la preuve de leur autorité en tant qu'interlocuteurs queer valables. Quoique je sois d'accord sur le fait que l'expérience de la marginalité puisse modifier la perception du sujet dans son approche du monde (en prenant pour acquis que ce dernier base sa perception sur la seule expérience du désir...), je me demande dans quelle mesure cette perception pourrait être représentable et pertinente dans le contexte de l'énonciation du discours queer, par exemple. Chacun peut voir dans le rapport homosexuel ce qu'il veut, mais il faut éviter que le discours queer ne devienne une expérience de thérapie de groupe digne d'être diffusée en après-midi... Je me réfère à Wittgenstein:

Das Wesentliche am privaten Erlebnis ist eigentlich nicht, daß jeder sein eigenes Exemplar besitzt, sondern, daß keiner weiß, ob der Andere auch dies hat, oder etwas anderes. Es wäre also die Annahme möglich -obwohl nicht verifizierbar- ein Teil der Menscheit habe eine Rotempfindung, ein anderer Teil eine andere.[2]/pp. 8-9/

Cette volonté de retranchement du discours queer à un espace qui ne serait accessible que par la confession presque entière de certains éléments de la sphère privée, en particulier ceux concernant le désir sexuel, se heurte à une impossibilité très bien décrite par Julia Kristeva dans une entrevue accordée au magazine littéraire Lire. La psychanalyste donnait son opinion sur la possibilité d'éclairer l'oeuvre de certains auteurs par une biographie qui étalerait les détails de leur vie sexuelle. (On pense par exemple à la biographie de Simone de Beauvoir par Deirdre Bair, où les aventures homosexuelles de Simone de Beauvoir sont décrites à grands renforts de détails et de témoignages inédits.) Toutefois,

... le galvaudage de la psychanalyse sous cette forme présente un danger: celui de faire croire que la sexualité peut se dire directement. C'est une naïveté de croire qu'il peut y avoir une transparence psychique.[3]

\section{L'homosexualisation de l'Histoire}


Le point de vue des deux intervenantes de la Colombie-britannique soustendait l'acceptation d'une essence homosexuelle, définie par certains critères relevant de l'analyse de cette construction culturelle. La communication de Matt Kuefler acceptait aussi d'emblée cette conception essentialiste dans son attaque contre L'Histoire de la sexualité de Foucault. Kuefler défend l'hypothèse de l'existence d'un groupe homosexuel reconnu comme tel avant le 19ème siècle, avant l'époque donc où, selon Foucault, l'homosexuel en tant que sujet médical et juridique aurait fait son entrée dans l'ordre du discours occidental. Cette vision historique de l'homosexuel, telle que défendue aussi par Gary Kinsman,[4] perçoit assez de similarité entre la construction sociale de l'homosexuel contemporain et celui de la Renaissance pour parler de Gay history. On pourrait parler de colonisation du passé et de transposition d'un modèle discursif contemporain à une situation où la marginalité n'était même pas une catégorie. L'usage de l'histoire de l'homosexualité, telle que présentée par Gary Kinsman, implique la notion de conservation de l'espace sexuel. Il est cependant nécessaire de se mettre en garde contre ce projet d'histoire gaie à la lumière d'une réflexion sur l'abus de l'histoire par d'autres groupes d'intérêt qui, quoique poursuivant des buts persuasifs complètement différents, ont aussi fait la preuve que l'on peut faire dire n'importe quoi à l'histoire dans le but de revendiquer la légitimité d'un mouvement idéologique contemporain. Pensons un instant à l'histoire allemande telle que revue et corrigée par les nazis; pensons aussi à l'histoire du Québec enseignée d'une manière différente selon la couleur du parti au pouvoir. Ces renvois à l'"histoire gaie", par exemple à certains cas de sodomie condamnés en NouvelleFrance, pour dénoncer une tradition hétérosexiste "canadienne" (les limitations à l'espace géographique canadien m'apparaissent aussi complètement impertinentes: elles rejoignent cette obsession de la création d'un espace à soi dans le but de justifier une essence queer) ne sont, selon moi, qu'un recyclage aveugle de faits qui, quoique sûrement véridiques, s'avèrent inutiles pour la cause de la libération des homosexuels dans le contexte social d'aujourd'hui. La création d'un espace historique queer, comme s'il s'agissait d'un groupe ethnique au même titre que les hispanoaméricains, cache mal l'intention d'inventer une essence gaie basée sur la ségrégation, dont plusieurs générations d'homosexuels ont souffert, et de tirer de l'expérience douloureuse (et autrement plus menaçante pour l'homosexuel du siècle dernier) la permission de revendiquer certains droits pour réparer le mal fait au cours des siècles. L'homosexuel contemporain doit assumer le fait qu'il est une construction discursive et que le refuge de la "minorité opprimée" n'est basée que sur un discours calqué sur celui d'un certain type de féminisme.

Voilà ce qui a été mis au clair par Jeannelle Savonna de l'Université de Toronto: la dette épistémologique dont les théories queer, gaies et lesbiennes sont redevables aux discours féministes essentialistes. Sa mise en garde contre les écueils qui pourraient nuire à la construction d'une essence queer me semblent en effet très pertinente. Elle remet en question la possibilité d'une union des discours féministes et queer par la différence même des lieux d'énonciation. On peut en effet s'interroger sur le désir 
d'une féministe, par exemple, de s'identifier au mouvement queer pour protéger justement l'intégrité de son espace. Si l'espace queer exprime le désir d'accueillir en son espace quiconque montre patte blanche[5] (par la subversion du genre, des normes sexuelles, etc.), il n'en est pas de même pour plusieurs féministes séparatistes qui ne voient pas d'un bon oeil l'utilisation de leurs paradigmes de revendication par un groupe comme les gais qui se réclamerait d'une essence similaire à la leur. C'est là où commence cette tendance à l'exclusion qui se poursuit jusque dans le passing, une manifestation de la vague politically correct dans l'institution. Le passing consiste, comme on peut s'en douter, à se donner l'autorité discursive en revendiquant une fausse identité lesbienne ou gaie. Que le phénomène soit répandu ou non dans l'institution nord-américaine n'est pas la question; dans les faits il s'agit d'entrer à tout prix dans la marginalité pour éviter de faire partie du discours dominant male hétérosexuel blanc. Le queer serait donc le groupe marginal le plus accessible puisque n'importe qui peut se revêtir de son essence en soumettant l'expérience personnelle comme preuve de son identité. Ce phénomène passager, témoin de la récupération par l'institution du pouvoir de la victime, prouve selon moi que les concepts d'espace et de positionnement du sujet sont extrêmement instables et qu'ils se limitent à un espace discursif nord-américain. De surcroît, ils s'avèrent très narcissiques puisqu'il totalisent l'expérience homosexuelle (et j'entends par là le contact, l'attirance et le désir homosexuel) à l'intérieur d'une construction discursive américaine culturellement limitée à la réalité urbaine occidentale. Un bon exemple de cette tendance globalisante seraient ces guides de voyage adressés à l'attention des homosexuels globe-trotteurs (Spartacus ou encore Odysseus) qui classent chaque pays par ordre alphabétique et qui dirigent le voyageur en quête de contacts avec ses "semblables" de par le vaste monde. Ainsi le Manitobain de passage à Casablanca saura vers quel sauna se diriger et il apprendra à quel Kaffeehaus viennois s'installer pour se trouver dans l'espace libre de son identité gai: le monde devient ainsi la banlieue de sa culture queer.

Autour des communications du colloque circulait aussi une atmosphère créée par des incidents intéressants, comme ces tracts dissimulés dans des morceaux de carton multicolores pliés en forme de triangle de sorte que l'on devait les briser pour les lire. Plusieurs photocopies de ce message avaient été déposées à des endroits très passants pour s'assurer d'un public nombreux. On y lisait:

\section{OUTING}

\section{ABUSE OF POWER}

\section{RACISM}




\author{
AND NOW...
}

APPROPRIATION OF CULTURAL SPACE

IT HAS COME TO OUR ATTENTION THAT A

PAID ACADEMIC FROM A WESTERN

UNIVERSITY HAS OUTED LESBIAN GRAD

STUDENTS AND NOW INVADES OUR QUEER

SITE.

FEEL SAFE? BROUGHT TO YOUR ATTENTION BY L.T.

Voilà qui frôle la théorie de la conspiration. On conçoit ici clairement l'usage de l'expression "espace culturel" en termes de propriété et de chasse-gardée d'une certaine classe d'individu. Une question se pose quant à savoir si l'auteur de ce message l'aurait quand même rédigé si l'academic en question n'avait pas été salarié, ce qui nous ramène à des motifs économiques. Voilà le genre d'abus auquel nous conduit le discours de la discrimination: il enlève toute crédibilité au sujet soucieux de fonctionner de manière intrègre en évitant de se servir de ce genre de message qui n'est en fin de compte que la répétition duouting qu'il dénonce. Est-ce qu'on peut justifier ce genre de règlement de compte au sein de l'institution? Peut-on continuer à barboter dans ces accusations, à pointer d'un doigt accusateur celui qui a osé nous appeler par le nom que nous nous sommes donnés? L'"invasion de l'espace queer" n'est pas une trouvaille de L.T.; il se laisse lire fréquemment dans le discours revendicateur.

\title{
Accès interdit
}


Gary Kinsman parle de invasion of our sexual space pour qualifier la fermeture forcée par la police de Toronto, au début des années 80, des saunas gais.Récemment, aux États-Unis, la controverse autour de la tentative du gouvernement Clinton de donner une place aux gais dans l'armée américaine est aussi un exemple de ce combat territorial. Si l'on examine le dernier exemple de plus près, on pourrait dire que c'est à partir du moment où l'acte sexuel est transposé comme identité sur l'individu qui le pratique que la discrimination commence, car qui peut nier l'activité homosexuelle au sein de l'armée américaine? De la même manière, qui peut ignorer la fébrile activité sexuelle qui régnait dans les ordres religieux masculins au Québec? Les organisations comme l'armée, les ordres religieux et autres groupes masculins hermétiques fonctionnent de la même manière que la communauté gaie urbaine nord-américaine telle qu'elle se donne à voir: une iconographie hyperdéveloppée (uniformes, gestuel, langage, toute la sémiotique du groupe) et un principe d'exclusion pour les sujets incompatibles avec ce système. Le coming out de l'homosexuel serait un rite de passage dans l'espace queer comparable aux voeux religieux ou à la rentrée dans l'organisation militaire. Ces trois discours ont, selon moi, des tendances similaires à créer leur propre histoire, à coloniser le passé et à organiser un système de valeurs basé sur une notion du territoire. De la caserne au village gai de la grande ville nord-américaine, l'uniforme et le discours (les codes linguistiques et non- linguistiques) confirment le droit au territoire. C'est en énonçant un espace essentialiste queer, queer en tant qu'espèce humaine, que nous ouvrons toutes grandes les portes à la discrimination. Je me demande à quel point le débat sur les gais dans l'armée américaine aurait pu prendre une tournure différente si l'on avait plutôt parlé du droit des militaires à s'adonner à des pratiques sexuelles diverses. Le choix des termes dans le contexte de telles revendications décide souvent de l'issue des négociations. On ne peut, en effet, faire accepter la notion d'espace gai et d'essence homosexuelle à des instances qui refusent l'existence et la complexité de ses constructions culturelles.

Le désir d'implanter en son territoire un système d'icônes constitue dans le discours gai une tendance commune qui s'est manifestée au colloque du New College à maintes reprises. La communication de Steve Maynard en est un exemple frappant. Ce dernier présentait une analyse des publicités de sous-vêtement qui, à son avis, conditionnent les critères de la beauté masculine dans la communauté gaie, critères dont les compagnies se serviraient à des fins commerciales. Son intention a semblé être de présenter cet état de choses comme un effet négatif du "clonage" de l'homosexuel en tant qu'objet de désir, et de dénoncer l'usage des photographies de jeunes David en sous-vêtement. Sa communication cependant, qui était illustrée par des diapositives de ces publicités (une à chaque 10 secondes...) devant un auditoire composé à $90 \%$ d'hommes (les quelques femmes présentes ayant quitté la salle après la troisième diapositive...) visiblement excités par la chose, s'est soldée par un spectacle plus intéressant que la dénonciation du médium. Faut-il conclure, de cet exemple, que l'essence gaie se réduirait à l'attirance physique et ne 
résisterait pas à une tentative de se définir comme groupe social ou culturel?

\section{Des Canadiens dans l'espace}

Andy Fabo, en reprenant l'argumentation de Susan Sontag sur le camp n'a pu selon moi éviter de tomber dans le piège /pp. 14-15/ que l'espace pose. Sa tentative d'importer la sensibilité camp dans le contexte artistique canadien s'est avérée un exemple d'anglo- centrisme effréné (ou de torontocentrisme, puisque nous nageons dans les néologismes et dans un imbroglio lexical inextricable!). Sa communication, intitulée "Notes from Camp Canada...", était illustrée par trois exemples tirés du milieu artistique torontois faisant un usage conscient du camp. Or, Susan Sontag définit le camp assez précisément comme un phénomène qui, bien sûr, est le plus souvent manifeste dans les milieux homosexuels urbains artistiques américains, mais qui néanmoins a surtout la caractéristique d'être inconscient et apolitique. Peut-on parler de camp canadien si, a priori, cette sensibilité est par définition un phénomène apolitique? Je crois que si la réponse à cette dernière question est positive, on peut facilement trouver des exemples de camp naïf partout à la télévision canadienne sans avoir à se restreindre à la culture du ghetto gai, surtout quand on sait que le camp a comme impératif de faire partie de la culture des masses (sinon, Fabo devrait faire preuve d'un peu plus de délicatesse pour les âmes outremétropole en appelant sa conférence Notes from Camp Toronto...) Enfin, mon but était moins d'argumenter sur la nature et la définition du camp que de donner un exemple de cette tendance à la recherche d'un espace commun, dans ce cas-ci d'un camp canadien, où le sujet se retrouve maître du discours en s'y plaçant au centre.

\section{Quitter l'espace}

Le discours gai nord-américain délimite son espace en tant que construction sociale à l'aide d'un système sémiotique proche de celui du théâtre, et se soumet volontairement à cette autorité iconographique qui le place dans un espace visuel précis. De là les termes de "sensibilité et écriture homosexuelles" (qui rappellent étrangement les concepts d'écriture féminine de Cixous...) et de "gay subculture". Le comportement sexuel ne serait qu'une donnée de cette essence très complexe pour les tenants de la théorie essentialiste, et pour les autres la condition unique et essentielle de l'homosexuel précédant toute dénomination. La question semble être: est-ce que le phénomène se laisse limiter à l'expérience, ou est-ce l'essence qui provoquerait l'expérience? Les récentes découvertes liant l'homosexualité 
masculine à l'héritage génétique n'expliquent pas l'absence de ce que nous appelons (à défaut d'une traduction qui rendrait justice au terme) a gay lifestyle dans les cultures islamiques, où le rapport homosexuel est fréquent mais ne saurait être défini en termes "d'espace culturel" puisque les hommes le pratiquant n'y rattachent pas tout l'attirail des valeurs culturelles associées au coming out américain.

Le projet queer consisterait à assimiler l'expérience individuelle au sein d'une large essence collective en se basant sur des paradigmes qui déterminent la validité de chaque expérience personnelle dans la sphère publique. Le queer serait le fourre-tout du discours de la différence, rayon du sexe. À l'aide de divers outils professionnels et selon les courants en vogue de la pensée universitaire, le queer représenterait un espace où il faut montrer patte blanche avant d'être admis en tant que sujet et objet du discours. Introduire l'acte sexuel ou l'attirance physique entre deux êtres, comme instrument théorique, consiste à les réduire ou à les abaisser au domaine des choses classées et hiérarchisées dans le monde selon des valeurs issues de l'expérience.

\section{Dans le lit de Marguerite}

Quel serait donc le lieu queer qui ne ferait partie d'aucun espace figé? La poésie et la littérature, où la fonction utilitaire et communicationnelle de la langue peut être évacuée et où le langage glisse en dehors de toute représentation possible, proposent une manière d'appréhender le comportement sexuel autrement que par la dénomination réductrice et limitante des catégories telles que gaie, lesbienne, bisexuelle (et autres minorités sexuelles, comme il m'a déjà été donné de lire dans un pamphlet publicitaire à l'intention d'un centre communautaire). Nous devenons ce que nous laissons le discours faire de nous. La métaphore spatiale nous pousse dans une catégorie molle de laquelle il est aisé de s'extraire puisqu'on y entre comme dans un moulin. Des oeuvres comme Les yeux bleus cheveux noirs de Marguerite Duras parviennent, par le langage littéraire, à rendre l'homosexualité omniprésente dans le texte sans la nommer une seule fois. Le film et le livre Les nuits fauves, de Cyril Collard, réussissent à extirper l'homosexuel de l'image du dandy dans laquelle la critique littéraire l'avait confiné. C'est en sortant de cette construction mentale qu'est l'espace queer, que nous arriverons à toucher au phénomène concret du désir de l'autre sexe et que nous éviterons une séparation épistémologique qui relèguerait les lectures queer aux oubliettes. Tenter de trouver une essence transcendante au comportement sexuel consisterait à séparer le corps de ce qu'il peut faire en dehors de l'homosexualité. Les études sur l'homoérotisme, en littérature, ont leur place au sein d'un corpus plus large que celui d'un cours de littérature féministe ou d'un cours de littérature gaie et lesbienne. L'enjeu est de reconsidérer l'importance de la position du sujet, 
d'annihiler les frontières de l'espace queer pour l'inscrire dans une politique plus large de l'écriture du corps, des sens et du désir. Il faut sortir de l'espace queer et amorcer une pensée du mouvement, loin des catégories statiques de l'histoire queer et loin de l'autorité discursive, sans revendiquer de spécificité au sein de l'institution au nom de l'expérience personnelle: sortir de ce psy-show pathétique que sont devenus ces colloques queer. Des efforts nombreux sont faits en ce sens. On pourrait dire que la communication de Mary Bryson et de Suzanne de Castell a amorcé un questionnement sur la possibilité de l'autorité discursive dans le discours du désir. Je crois qu'il faut repenser les concepts d'espace et d'expérience afin de donner aux études queer la crédibilité et l'attention qu'elles méritent au sein de l'institution. Il nous faut élaborer de nouveaux paradigmes qui respecteraient l'unicité de son discours (sans laquelle un discours n'existerait pas), en parlant plutôt de politiques et de poétiques du désir pour éviter de se cantonner dans une marginalité factice et propice à la discrimination.

\section{Eric Dupont}

\section{Département de Littérature comparée}

\section{Université de Montréal}

Surface Page d'Acceuil/Home Page

\section{Bibliographie sélective:}

Blanchot, Maurice. L'espace littéraire. Paris: Gallimard, coll. Essais, 1955.

Duras, Marguerite. Les yeux bleus cheveux noirs. Paris: Minuit, 1986. . La pute de la côte normande. Paris: Minuit, 1986. . L'été 80. Paris: Minuit, 1980.

Foucault, Michel. La volonté de savoir. Paris: Gallimard, 1976.

Greenberg, David. The construction of Homosexuality. Chicago, 1988. 
Herlihy, David. "Is there a history of Sexuality?" In History and Theory 28 (1989): 257-74.

Kinsman, Gary. The Regulation of Desire: Sexuality in Canada. Montréal, 1987.

Kristeva, Julia. "Peut-on dire la sexualité?" In Lire 213 (juin 1993).

Mohr, Richard. "The Thing of It Is: Some Problems with Models for the Social Construction of Homosexuality." Ch.7 in Gay Ideas: Outing and Other Controversies. New York, 1992.

Wittgenstein, Ludwig. Philosophische Untersuchungen. Oxford: Basil Blackwell, 1967.

[1]Voir le compte-rendu de Cristophe Park dans ce volume, \#20.

[2]Wittgenstein, Ludwig, Philosophische Untersuchungen, Basil Blackwell, Oxford, 1967, p.95.

L'essentiel à propos de l'expérience personnelle n'est en fait pas que chaque personne possède son propre exemplaire, mais que personne ne sait si l'autre a aussi cela, ou autre chose. La supposition serait donc possible quoique qu'invérifiable qu'une partie de l'humanité aurait une perception du rouge et qu'une autre partie en aurait une autre. [ma traduction]

[3] Julia Kristeva, "Peut-on dire la sexualité?" in Lire 213 (juin 1993): 22.

[4]Gary Kinsman, The Regulation of Desire, Sexuality in Canada (Montréal: Black Rose Books, 1987).

[5]"Montrer patte blanche" réfère au conte dans lequel une brebis, devant s'absenter de la bergerie pour la journée dit à ses agneaux de n'ouvrir la porte qu'à ce qui pourrait montrer patte blanche à travers un orifice taillé dans la porte. Au courant de cet avertissement, le loup réussit à s'introduire dans la bergerie en roulant sa patte dans la farine. Les agneaux n'y voient que du feu et laissent le prédateur entrer. Le loup serait-il un maître du passing? 